Methods A total of 33 blood specimens collected from 16 patients presenting with a syphilitic ulcer were analysed. The specimens were tested employing: Rapid Plasma Reagin; Treponema Pallidum Particle Agglutination (TPPA); recomLine Treponema IgM (Mikrogen Diagnostik); recomWell Treponema IgM (Mikrogen Diagnostik); Anti-Treponema pallidum ELISA -IgM (Euroimmun). Ulcer specimens were tested by qPCR. A specimen was considered positive for anti-Tp IgM when both TPPA and IgM, obtained with any of the assays, were positive.

Specimens containing antibodies against Epstein Barr Virus, Leptospira sp, Borrelia sp, Plasmodium sp, Herpes Simplex Virus 1 and 2 and specimens from pregnant women were tested with the three IgM assays to determine cross reactivity.

Results Anti-Tp IgM was found in 22/33 specimens. The recomLine assay detected 17/22 (77\%), the recomWell 15/22 (68\%) and the Euroimmun 14/22 (64\%) positive specimens. None of the three assays provided false positive results. Borderline results were obtained with the recomLine $(\mathrm{N}=6)$ and the recomWell $(\mathrm{N}=2)$ assay. Considering the borderline results as positive increased the detection rate to $95 \%$ for the recomLine and to $72 \%$ for the recomWell. However, two and one false positive result was then obtained with the recomLine and recomWell assay, respectively. Tp DNA was detected in 13/16 (81\%) ulcer specimens. The Euroimmun assay cross reacted with malaria antibodies in one sample.

Conclusions None of the three assays showed to be highly sensitive. Surprisingly, the highest sensitivity was obtained with the recomLine assay. The sensitivity improved by defining the borderline results as positive but decreased the specificity.

Disclosure of interest The IgM assays were kindly provided by the two companies, Euroimmun and Mikrogen Diagnostik. No other funding was received for this work.

\section{P05.04 ANTIMICROBIAL SUSCEPTIBILITIES OF PERSONS WITH GONORRHOEA AT MULTIPLE SITES ARE ACCURATELY REFLECTED BY UROGENITAL SPECIMENS}

P Dixon*, B Van Der Pol, J Swerdlow, S Cammarata, E Sun, EW Hook. University of Alabama at Birmingham, Birmingham AL and Melinta Therapeutics New Haven, CT, USA

\subsection{6/sextrans-2015-052270.290}

Background Surveillance for gonococcal (GC) antimicrobial resistance is focused on susceptibilities of urogenital GC isolates. The ability of surveillance programs utilising only urogenital specimens to detect resistance in communitites could theoretically be compromised if extragenital (i.e. pharyngeal or rectal) GC infectons were more often resistant. We evaluated how GC antimicrobial susceptibilities at extragenital sites varied when compared to contemporaneously isolated urogenital infections. Methods We determined GC agar dilution MICs for ceftriaxone, cefixime, ciprofloxacin and azithromycin in isolates from 57 participants with multisite infections in a recent multicenter GC treatment trial.

Results Extragenital infections were more common among men with male partners (MSM - 23 [19\%] of 118) and women (21 [18\%] of 118), than men with female partners (MSW- 13 [8\%] of 169) In this study all extragenital infections in MSWwere pharyngeal while pharyngeal isolates comprised about half of extragenital infections from MSM (12\[52\%] of 23) and women (14[67\%] of 21, respectively).

MICs were meaningfully different (2 or more dilutions) between urogenital and extragenital sites in $9(16 \%)$ of 57 persons (14[6\%] of 228 comparisons) with multiple site infections. Ceftriaxone or cefixime MICs varied between urogenital and extragenital sites in just 2 participants while MICs for ciprofloxacin and azithromycin varied in 3 and 7 participants, respectively. Only urogenital isolates from women and MSM had significantly elevated MICs to azithromycin when patients with infections at multiple sites were compared to those with only urogenital infection. There was no consistent pattern to these differences; in 5 urogenital MICs were greater than extragenital sites while in the remaining 4 participants, extragenital MICs were greater than for urogenital sites.

Conclusions MICs for urogenital sites of infection most often reflect MICs of extragenital isolates in persons with multiple simultaneous sites of infection and are suitable for surveillance for antimicrobial resistance.

Disclosure of interest Funding for this study was provided by Melinta Therapeutics.

\section{P05.05 NEISSERIA GONORRHOEAE IN INDONESIA: PREVALENCE AND ANTIMICROBIAL SUSCEPTIBILITY AMONG STI CLINICS PATIENTS IN JAKARTA, YOGYAKARTA AND DENPASAR}

1,2,3PY Hananta*, ${ }^{3} \mathrm{AP}$ van Dam, ${ }^{3} \mathrm{SM}$ Bruisten, ${ }^{2,3} \mathrm{MF}$ Schim van der Loeff, ${ }^{1} \mathrm{H}$ Soebono, ${ }^{2,3} \mathrm{HJC}$ de Vries. ${ }^{1}$ Department of Dermatology and Venereology Faculty of Medicine Universitas Gadjah Mada, Yogyakarta, Indonesia; ${ }^{2}$ Academic Medical Center Universiteit Van Amsterdam, The Netherlands; ${ }^{3}$ Public Health Service (GGD) of Amsterdam, The Netherlands

\subsection{6/sextrans-2015-052270.291}

Introduction In Indonesia, syndromic management is used for diagnosis and treatment of sexually transmitted infections (STIs), but this approach does not provide accurate data to assess the epidemiology of gonorrhoea and antimicrobial susceptibility of circulating Neisseria gonorrhoeae strains. We studied gonorrhoea prevalence and antimicrobial resistance of N. gonorrhoeae among patients of STI clinics in 3 large cities in Indonesia.

Methods In 2014, urogenital swabs were obtained from sexually active adults, mainly female sex workers, men who have sex with men, and male-to-female transgenders attending STI clinics in Jakarta, Yogyakarta, and Denpasar. In some patients additional rectal and pharyngeal swabs were taken. Diagnosis of N. gonorrhoeae infection was established by real time PCR targeting Opa genes. Culture isolates of N. gonorrhoeae were tested for antimicrobial susceptibility against doxycyclin, ciprofloxacin, azithromycin, ceftriaxone and cefixime by E-test $^{\mathrm{TM}}$ using EUCAST breakpoints.

Results We included 764 participants: 346 (45.3\%) males, 321 (42.0\%) females and 97 (12.7\%) transgenders (median ages 27, 30 , and 38 years). In total, 800 samples were collected: 443 urethral, 321 cervical, 23 rectal, and 13 pharyngeal swabs. In 215 (28.1\%) participants N. gonorrhoeae infection was detected by PCR. Prevalence varied significantly by gender $(26.0 \%$ in males, $32.1 \%$ in females, and $22.7 \%$ in transgenders, $\mathrm{p}=0.04)$; and varied significantly by city: $14.5 \%$ in Denpasar, $31.5 \%$ in Jakarta and $27.5 \%$ in Yogyakarta $(\mathrm{p}=0.015)$.

Of 77 isolates tested for antimicrobial susceptibility, all were sensitive to azithromycin, ceftriaxone, and cefixime. In contrast, resistance to doxycyclin (98.7\%) and ciprofloxacin (100\%) was very common.

Conclusion This is the first study since 2004 describing the epidemiology of gonorrhoea and antimicrobial resistant strains in Indonesia. Prevalence of gonorrhoea is very high among STI 